\title{
Determining the Optimal Type of Non-Smooth Intersection Using Particle Mass Algorithm (PSO)
}

\author{
Seyed mohammad sadegh nasiri \\ Civil engineer beyza Islamic azad university shiraz iran
}

\begin{abstract}
Nowadays, with increasing number of vehicles, the intersections of the same level are in many problems. There are different ways to solve this problem, which is now converting the intersection of the intersection to the unequal intersection of the engineers' attention. But a model that takes into account different factors of the intersection is not in an optimal level. In this research, we have tried to provide a model for choosing the optimal type of intersecting intersection with consideration of effective factors. The intersections in this study are directional intersections, full cloying, semi-clover, single-point and rhombus simulated using the AIMSUN software. The parameters considered for each intersection are the amount of air pollutants produced, fuel consumption, and travel time. And the value of the above parameters is derived from the outputs of the AIMSUN software, then all of them are converted to cost. Subsequently, using the massive particle algorithm (PSO) in the following of the study was used, and this algorithm is used to calculate traffic volumes of 1000 , 3500 and 6500 vehicles per an hour, respectively, in intersections of directional, rhomboid and single-pronged as an intersection, the optimal non-level is presented.
\end{abstract}

Keywords: Non- intersection crosses, Prioritization, Mass Particle Algorithm (PSO)

DOI: $10.7176 / \mathrm{CER} / 11-6-03$

Publication date:July $31^{\text {st }} 2019$

\section{Introduction}

With increasing population and consequent growth in travel demand, transport managers are faced with the challenge of the safe and efficient transportation of man and goods due to land and land constraints, especially in the highways and streets in urban and out of cities, where there is no possibility of increasing capacity, congestion increases travel time and reduces safety and, as a result, unbearable conditions for the driver. In recent years, the emergence of uneven level intersections as a promising solution to effective intersection management has been considered with regard to existing infrastructures. The use of this conversion has potential benefits, such as improved time Travel, reducing environmental pollutants, long-term capital savings and reducing fuel consumption. In 1972, Walters and Ingram evaluated the peak hourly capacity of each of the options using a linear model. A member of the intersection affected by the maximum volume and the more critical conditions is identified, and in the next step, the capacity is increased to produce the appropriate model. In 1973, Molynazi and Sutherli compared non-level intersections through the level of service, safety, flexibility of length and number of lines of cross-border areas, travel time, and social factors on non-intersecting intersections. In 1977, Smith and Garber compared the two intersections of rhomboid and single-stranded junctions based on the immunity parameter.

\section{Statement of the problem}

One of the solutions that engineers see to improve the current status of the intersection is to modify the geometric design, apply traffic constraints, etc., which are in line with low cost solutions. If these solutions can not solve the intersection traffic problem, then another solution, which is the same as the intersection, is considered. Due to the wide variation in the types of intersections and the scope of each of them, as well as the different economic costs, there are different options for choosing the type of intersection. In our country, when an intersection of an intersection is converted to a cross intersection, there are no criteria for choosing the optimal intersection of unevenness. The existence of a model that can provide factors such as traffic, economic, and environmental factors in a general framework for optimal selection of non-interconnected crosses are one of the basic needs of the community of engineers. As a result, it is necessary to develop a model for the design, implementation, and management of this model in order to be able to be used as a bachelor's judgment as well as a guide. In the foregoing article, using data Traffic and simulation software, five non-level intersections were simulated and placed under different traffic loads. Finally, simulation software outputs, equations for the desired parameters These include travel time, environmental pollutants and fuel consumption. Then, using these equations and an optimal algorithm massive particles to solve the problem (Hessami, 2009).

\section{Research background}

Aryan Amir Amjadi's method for non-level intersection crosses

In this paper, in the first stage, after selecting the software AIMSUN, all non-dimensional intersections of 
rhizome, single-strand, directional, full clover and semi-clover are modeled according to the specific shell in the software. In the following, the crosses are modeled under several different traffic scenarios for different traffic volumes, and each non-level intersection is simulated in the software, and after calibration of the software, which according to the field data in the Propagation Highway has been obtained. The outputs of the software are extracted. The parameters that are investigated in this research and are entered in the equations are: The amount of traffic, the amount of air pollutants, fuel consumption, travel time, cost of construction and safety. In the following, by using the AIMSUN software outputs for the parameters, the equation is based on the amount of left-turn volumes which have a decisive role in the operation of non-intersecting intersections. In the final stage, all parameters are converted to the rial cost, and therefore the output of the equations for each intersection is the cost of the intersection, which, as a result of a cross, which has a lower cost, can be introduced electively as a non - intersection cross. (Ahangari, 2005).

For weighting the parameters, using the questionnaires prepared by the experts and professionals of transportation and traffic as well as university professors and using the AHP method, the mentioned parameters were weighted. After importing the matrices in the expertchoice11 software and analyzing them by the software, geometric averaging of the final weights of the parameters of pollution, fuel consumption, travel time, space required, construction cost is obtained.

After obtaining the weight of the parameters for writing the total cost equation, each of the parameters of pollution, travel time, fuel, space required, construction cost in the weight obtained from the Expert choice 11 software is multiplied. As a result until this stage, we tried to apply the weight of each of the parameters under consideration. In the next step, in order to fit the unit, these parameters were combined to sum them together, multiplying all the parameters in the calculated costs by different methods, so at this stage, for each of the five types of non-intersections crosses, converted parameters to cost were summed together.

As a result, five main equations for the cost of the total of five non- intersecting crosses are obtained, in which the average volume of traffic to the left of the intersection, the number of crashes at the intersection, the area and the available space for the construction of the intersection of the non-level and the regional case $\mathrm{A}$ comment in Tehran is to construct the intersection of the non-equal inputs of the equation. Finally, these 5 equations, by entering the above parameters into 5 general costs for single-point-rhizosphere-directional-fulllength and semi-oblique shoots. An intersection with the lowest cost will be introduced as the optimal and selected intersection.

In view of the discussions and the results obtained from the equations, the following results are obtained from this research:

1) With increasing weight of the air pollution, directional crosse and clover are high priorities, respectively. Also, diamond crosses and single-junction crosses have the least priority (Amir Amjadis, 2011).

(2) As the diagrams show, due to the presence of a lamp at the diamond crosses and one-intersecting crosses, as well as high interference in the left and right turn movements, these intersections have the highest latency and pollution rates, and thus increasing the importance of travel time and air pollution in choosing a type of nonintersecting crosses, these crosses have a lower priority selection (Akhbari, 2008).

3) In this research, for the first time, a general indicator for choosing the optimal non-level intersection crosses based on traffic parameters, air pollution, cost of construction, fuel consumption and safety were present in one equation in the first place. 
Table 1: Research background

\begin{tabular}{|c|c|c|c|}
\hline year & Disadvantages & Method & Subject \\
\hline 1972 & $\begin{array}{l}\text { The linearity of the model used } \\
\text { to select the intersection } \\
\text { between user benefit and } \\
\text { intersection capacity }\end{array}$ & $\begin{array}{l}\text { A member of the intersection, which is affected by } \\
\text { the maximum volume and the more critical } \\
\text { conditions, is identified and, in the next step, } \\
\text { increases the capacity to produce the appropriate } \\
\text { model (Hasanpour, 2011). }\end{array}$ & $\begin{array}{l}\text { Watteleworth \& } \\
\text { Ingram }\end{array}$ \\
\hline 1973 & $\begin{array}{l}\text { Consider an arbitrary number } \\
\text { for each criterion }\end{array}$ & $\begin{array}{l}\text { Comparison through service level, safety, elasticity } \\
\text { of length and number of lines of cross-border areas, } \\
\text { travel time and social factors }\end{array}$ & $\begin{array}{l}\text { Mullinazzi \& } \\
\text { Satterly }\end{array}$ \\
\hline 1977 & $\begin{array}{l}\text { Limited application for two } \\
\text { single-point rhomboid and } \\
\text { intersecting junctions } \\
\end{array}$ & $\begin{array}{c}\text { Based on the immune parameters and the mode of } \\
\text { operation of the lumens and one-intersecting } \\
\text { intersections are compared. }\end{array}$ & Smith \& Garber \\
\hline 2009 & $\begin{array}{l}\text { The AHP method is not } \\
\text { suitable for interdependent } \\
\text { parameters (Eberhart \& } \\
\text { (Kennedy, 1995) }\end{array}$ & $\begin{array}{l}\text { Use the AHP method to select the best non-level } \\
\text { intersection }\end{array}$ & Zhou \& Liu \\
\hline 1387 & $\begin{array}{l}\text { Not considering different } \\
\text { parameters }\end{array}$ & $\begin{array}{l}\text { Provide an algorithm for selecting the optimal type } \\
\text { of non-intersecting intersection }\end{array}$ & $\begin{array}{l}\text { Seyed Kamal } \\
\text { Seyed Hossein }\end{array}$ \\
\hline 1390 & $\begin{array}{l}\text { Using hierarchical analysis and } \\
\text { not presenting an algorithm to } \\
\text { select an intersection } \\
\text { (Talukder, 2011) }\end{array}$ & $\begin{array}{l}\text { Prioritizing factors affecting the selection of non- } \\
\text { intersecting intersections }\end{array}$ & $\begin{array}{c}\text { Shahab } \\
\text { Hassanpour }\end{array}$ \\
\hline 1390 & $\begin{array}{l}\text { Investigating a particular type } \\
\text { of non-dimensional } \\
\text { intersections (directional) }\end{array}$ & $\begin{array}{l}\text { Evaluation of general configuration of left-handers } \\
\text { in non-level intersections using hierarchical } \\
\text { analysis method }\end{array}$ & Meysam Razi \\
\hline 1390 & $\begin{array}{l}\text { The AHP method is not } \\
\text { suitable for interdependent } \\
\text { parameters (Zhou \& Huang, } \\
\text {.2009) }\end{array}$ & $\begin{array}{l}\text { Using different parameters and applying the AHP } \\
\text { method }\end{array}$ & $\begin{array}{l}\text { Arian Amir } \\
\text { Amjadi }\end{array}$ \\
\hline
\end{tabular}

\section{Research Methodology}

The research process is that, at first, the reason for choosing the software for simulation of crosses (IESman software) is expressed, and then this software is described in detail, then about the selection of parameters Effective on choosing the optimal type of intersecting non-dimensional objects will be discussed and using effective decision parameters, effective parameters will be determined. In the following, we introduce the selected and effective parameters for choosing the optimal type of intersecting intersections. In order to model the problem, all the parameters selected have the same unit so each parameter is in a special way and Then, the method of modeling non-intersection crosses are investigated. Subsequently, we describe the massive paramagnetic particle algorithm and how it is used in this paper. Not be 


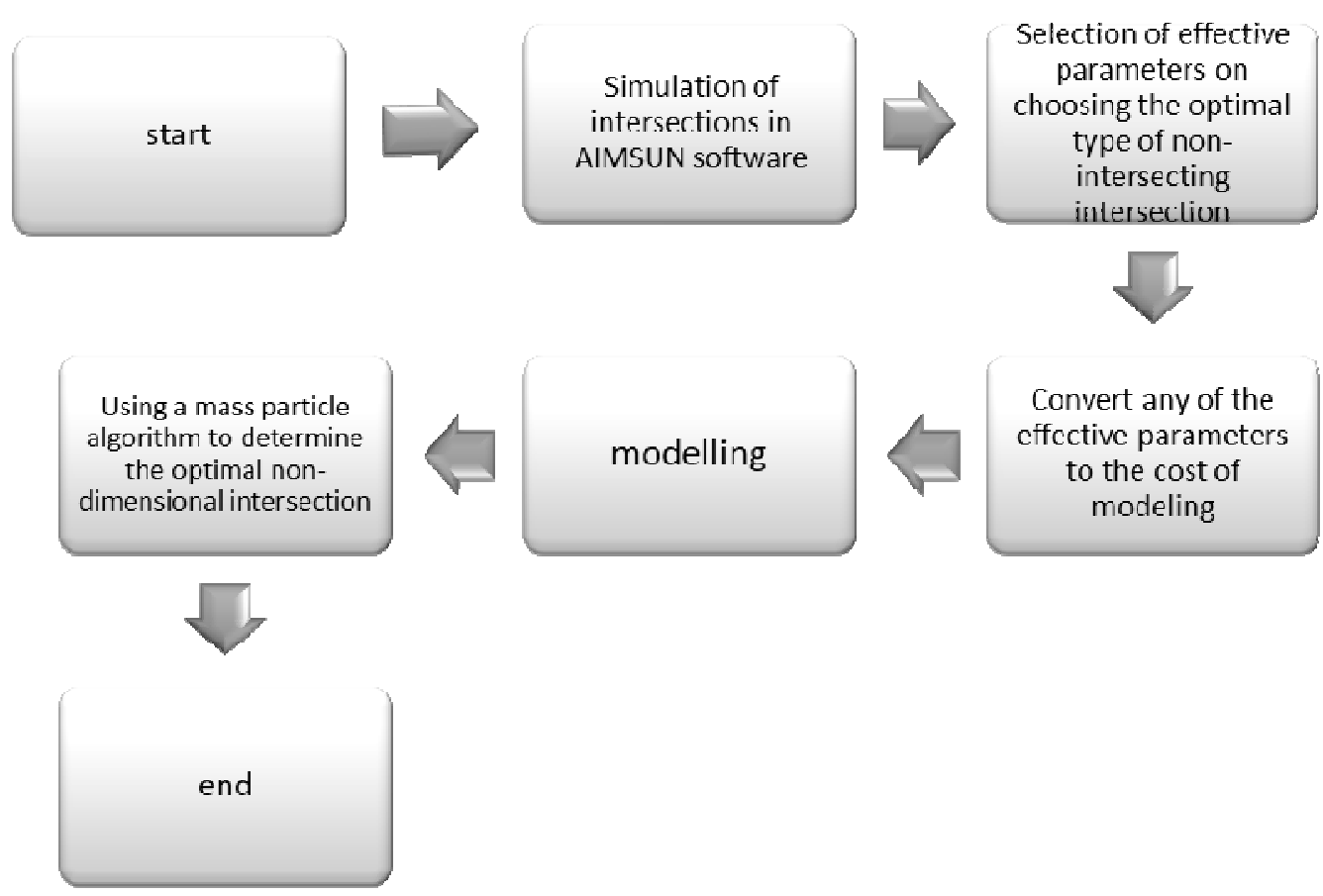

\section{Research findings:}

In the method, the priority is given to three factors: first, the parameters that are related to the cost, which include the amount of space needed for the construction of the non-intersection crosses, the cost of construction, etc., the second factor is the profit- Which includes travel time, fuel consumption, and air pollutants production, and the third factor is related to the perimeter of the intersecting intersection, which includes access points, outbreak facilities and non-intersecting crosses position in relation to other crosses. .

The prioritization with Dimthel model has been done for the three above-mentioned factors and five of the above-mentioned steps have been taken in this regard. Finally, the following graph has been obtained that the effect and effect of each factor Examples are:

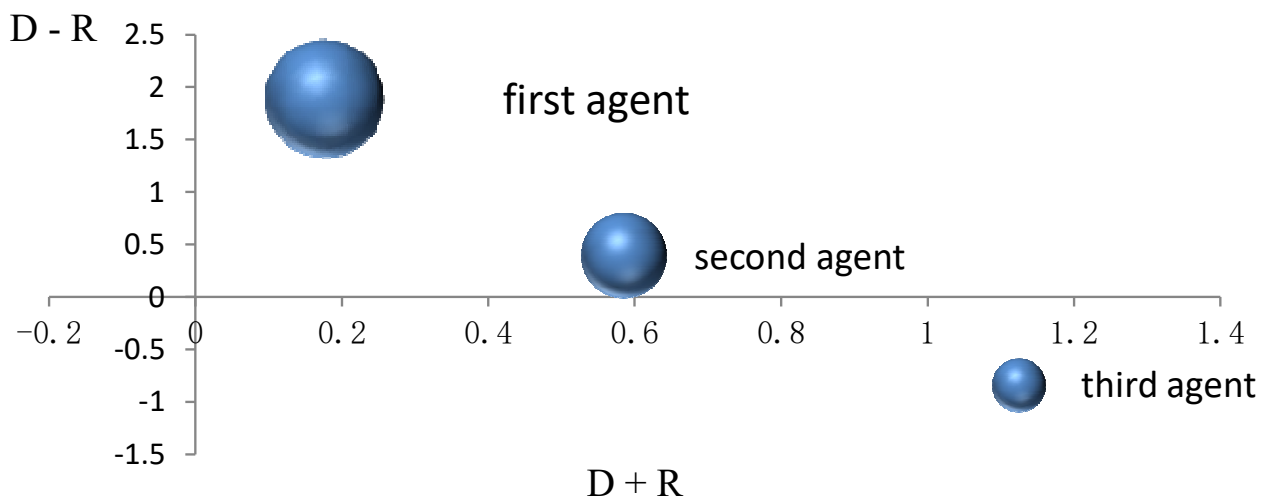

According to Figure 2, which shows the effect of three factors on the choice of the optimal type of nonintersection crosses, the first factor has the highest priority, the second factor has a moderate priority and the third factor has the lowest priority.

Artificial sources:

Synthetic sources have divided the emissions of air pollutants into stationary and mobile groups. The fixed group includes industries, power plants and commercial and residential centers, and includes moving vehicles from motorcycles to aircraft and ships. The pollution caused by the transportation sector due to unplanned urbanization is as important as industrial pollution. Contamination from transport and vehicles in the form of exhaust gases, suspended particles, sound, and so on. This pollution will be minimized by adopting national, regional and urban planning methods and the use of appropriate automobiles and fuels with the application of 

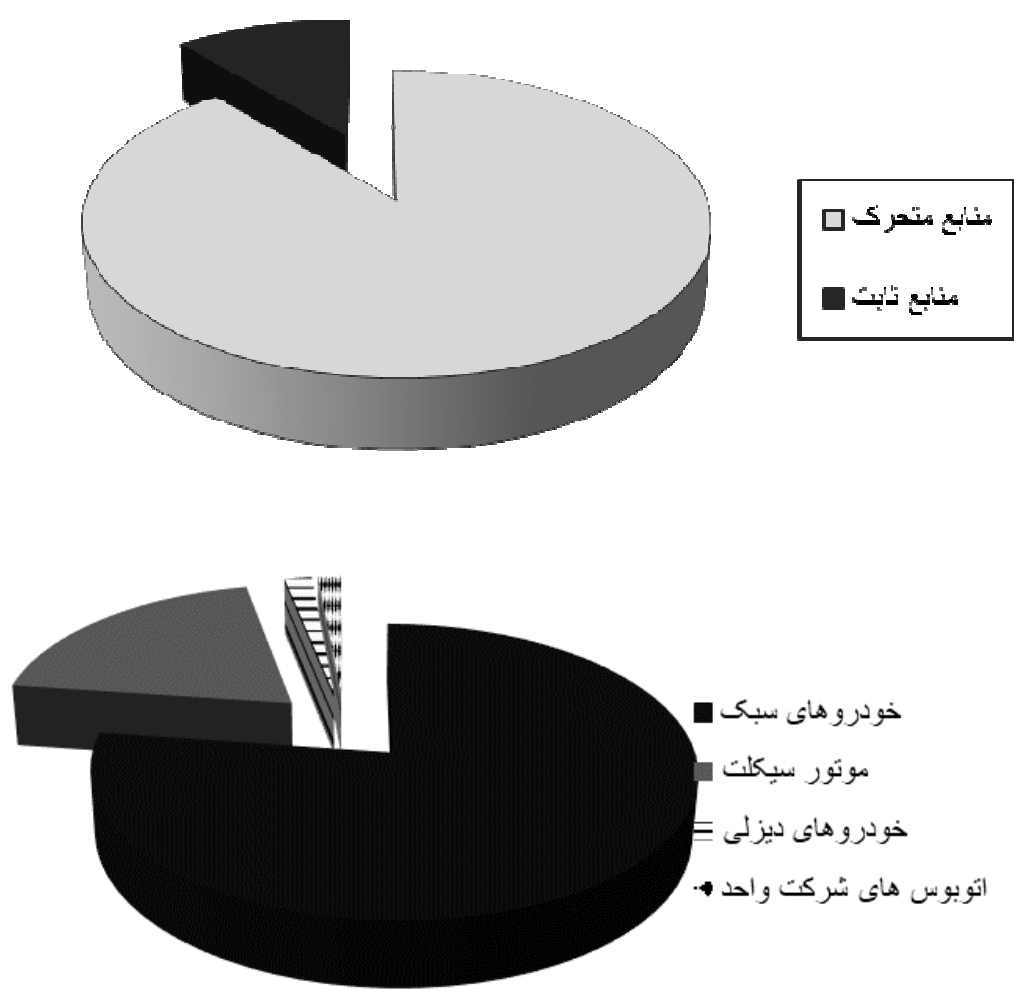

Figure 4: The contribution of different groups of transportation systems to pollution from migratory sources in Tehran air [8]

\section{Discussion and Conclusion}

In this article, important and significant points in the field of optimal selection of non-intersecting crosses have been discussed so that the use of meta-curiosity methods in the field of artificial intelligence algorithms can be used to achieve the objectives of the optimal selection of intersections were non-level. Therefore, in this paper, through the study of the technical aspects of artificial intelligence, the function of one of the most famous algorithms, namely the paramagnetic mass meta-algorithm, was depicted and based on the output data of this method, it was determined that in the state When the various traffic factors in the form of three equations of travel time, the amount of pollution and fuel consumption cause behavioral change, it can not be traced to planning based on traditional methods and there should be methods that can understand and analyze some very complex and complicated relationships.

Therefore, it can be argued that using artificial intelligence methods can be used as a tool for coping with the complexity of the multiplicity and diversity of non-intersections crosses and the optimal choice of them considers based on the models in the sources of interest. However, since the discussion of economic and social capital can be of great importance, the proposed tool can not only recognize the optimal option, but it can also help speed up the presentation of various analyzes. Given that even the smallest error can lead to the loss of huge capital, it is necessary to improve the efficiency of the proposed algorithms as much as possible. In this regard, the massive particle algorithm or movement of the bird group used in this paper has been satisfactory with good performance, and it can be stated that since the problem is more complicated by increasing the dimensions Therefore, not only this method can be suitable, but it can also function properly in dealing with this complexity. As the diagrams presented in this chapter are well presented, it can be noted that the performance of mass particle algorithm has been able to function properly. Most research on the comparison of mass particle algorithms and concepts also confirmed that the mass particle algorithm can be applied well in the problems that need to be optimized for several solutions simultaneously, which is also discussed in this paper. Using the mass particle algorithm, the following results are obtained:

1) In a traffic volume of 1,000 vehicles per hour, non-intersection directional crosses 
2) In the traffic volume of 3500 vehicles per hour, the diamond non-intersection crosses

3 ) In the traffic volume of 6,500 vehicles per hour, a single point non-intersection crosses

They were selected as the non-optimal intersection.

Offers

Using different methods, especially categorization methods, in combination with artificial intelligence algorithms such as:

$\square$ Genetic Algorithm

$\square$ Fuzzy systems

$\square$ Adaptive Fuzzy Neural Network

It is recommended to choose the optimal type of non-intersecting intersection.

The use of methods such as the adaptive fuzzy neural network is emphasized, because such methods can not only understand traffic information, including traffic volume, but also are capable of varying dimensions, such as the impact of other conditions, indicators and attributes The importance of every city or neighborhood as well as important management decisions are well understood.

\section{References}

1- Forging, Hamed, 2005, "Comparison of the Results of Different Methods for Estimating the Value of Travel Time", Journal of Transportation, No. 2.

2- Akhbari, Kamran, 2008, "Investigation of effective factors, including safety in the choice of non-level intersection approach", Eighth Iranian Conference on Transport and Traffic Engineering.

3. Amiramjadi , Arian,1390, "Choosing the Optimal Type of Non-Level Crossing in Intraregular Networks", Master's thesis, Khaje Nasir-Al-Din Tusi University of Technology, Faculty of Civil Engineering.

4- Hesami, Zohreh, and Wallisadeh Mihaji, Farnaz, 1389, "Social Costs Caused by Air Pollution in the Public Transportation Section", Experts of the Environment and Sustainable Development Department of Tehran Municipality.

5- Hassanpour, Shahab, 1390, "Prioritizing the factors affecting the selection of non-urban interurban options", 11th International Conference on Transport and Traffic Engineering.

6-Urban, 11th International Conference on Transport and Traffic Engineering. Eberhart R, Kennedy J,1995," Particle Swarm Optimization", Bureau of Labor Statistics Washington..

7-Zhou Q, Huang W and Zhang Y, 2009," Identifying critical success factors in emergency management using a DEMATEL method", school of management, huazhong university of science and technology,china.

8-Talukder S,2011," Mathematical Modelling and Applications of Particle Swarm Optimization", Thesis of master of science, Blekinge Institute of Technology.

9-Tanjima P, Ulf-G Gerdtham and Hampus C.L, 2008 " Societal costs of air pollution-related health hazards: A review of methods and results" 